\title{
Pilot-assisted Opportunistic User Scheduling for Wireless Multi-cell Networks
}

\author{
Hamed Farhadi, Hadi Ghauch, and Mikael Skoglund \\ Communication Theory Laboratory, School of Electrical Engineering \\ KTH Royal Institute of Technology, Stockholm, Sweden \\ Email: \{farhadih, ghauch, skoglund\}@kth.se
}

\begin{abstract}
We consider downlink transmission in multi-cell wireless networks where in each cell one base station is serving multiple mobile terminals. There is no a priori channel state information (CSI) available at base stations and mobile terminals. We propose a low-complexity pilot-assisted opportunistic user scheduling (PAOUS) scheme. The proposed scheme operates in four subsequent phases: channel training; feedback transmission; user scheduling; and data transmission. We deploy an orthogonal pilot-assisted channel training scheme for acquiring CSI at mobile terminals. Consequently, each mobile terminal obtains a noisy estimation of the corresponding local CSI (i.e. channel gains from base stations to the mobile terminal). Then, it makes a local decision based on the estimated channel gains of the interfering links (i.e. the links between base stations in neighboring cells and the mobile terminal) and sends a one-bit feedback signal to the base station of the corresponding cell. Each base station schedules one mobile terminal for communication. We compute the achievable rate region and the achievable degrees of freedom (DoF) of the proposed transmission scheme. Our results show that in a multi-cell network with $K$ base stations and coherence time $T$, the total DoF $K_{\mathrm{opt}}\left(1-\frac{K_{\mathrm{opt}}}{T}\right)$ is achievable given that the number of mobile terminals in each cell scales proportional to signal-to-noise-ratio. Since limited radio resources are available, only a subset of base stations should be activated, where the optimum number of active base stations is $K_{\text {opt }}=\min \left\{K, \frac{T}{2}\right\}$. This recommends that in large networks $\left(K>\frac{T}{2}\right)$, select only a subset of the base stations to be active and perform the PAOUS scheme within the cells associated to these base stations. Our results reveal that, even with single antenna at base stations and no a priori CSI at terminals, a non-trivial DoF gain can be achieved. We also investigate the power allocation between channel training and data transmission phases. Our study shows that in large networks (many base stations) more power should be allocated to channel training while in dense networks (many mobile terminals in each cell) more power should be allocated for data transmission.
\end{abstract}

\section{INTRODUCTION}

It has been provisioned that one of the most typical scenarios in $5 \mathrm{G}$ communications systems will be to deliver an exponentially increasing demand for data rate, in ultra dense deployments: such communication scenarios are characterized by a high data rate requirement that needs to be sustained, irrespective of the harsh urban propagation scenarios [1]. Moreover, the relatively high user density in such settings, implies that channel training and feedback overhead is major challenge. As a result, spectrally efficient transmission techniques with low-overhead, are much desired.

In order to enhance spectral efficiency in multi-user communication scenarios, the time-varying characteristics of wire- less transmission medium can be effectively exploited to opportunistically serve users that exhibit appropriate channel conditions. Several opportunistic transmission schemes have been developed including opportunistic scheduling [2]-[5], opportunistic beamforming [6], random beamforming [7], and opportunistic interference alignment [8]-[11]. These transmission schemes have been investigated in several cellular communication scenarios. The early opportunistic schemes have been mainly designed for exploiting multi-user diversity in single-cell communication scenarios (e.g. [2]-[4], [6], [7]). Recently, it has been shown that opportunistic transmission schemes can also mitigate inter-cell interference and thus achieve multiplexing gain in multi-cell communication scenarios (e.g. [8]-[11]).

The aforementioned schemes usually require certain channel state information (CSI) to be known at base stations and mobile terminals. For instance, the proposed schemes in [2][11] require perfect CSI to be a priori available at mobile terminals. Furthermore, [2], [3], [8] require base stations to perfectly know CSI, and [4]-[7], [9]-[11] need only quantized CSI to be available at base stations. However, acquiring CSI is a challenging problem in practice and base station and mobile terminals can attain only imperfect CSI. In particular, in dense communication systems there are many users and only limited radio resources are available for channel training and feedback transmission. Therefore, low-complexity channel training and feedback transmission schemes are desired that efficiently utilize available radio resources. In practice, CSI is not a priori available at mobile terminals and they may obtain only noisy estimation of CSI via channel training schemes. The impact of this on system performance is twofold: base stations need to allocate part of their radio resources for channel training and consequently less resources will be available for data transmission; and imperfect CSI may cause imperfect scheduling and erroneous decoding at mobile terminals and degrade the performance of opportunistic transmission schemes. The performance limits of opportunistic transmission with no a priori CSI at mobile terminals has been less known.

We consider a dense cellular communication scenario in which there is one base station in each cell serving a large number of mobile terminals with no a priori CSI available at terminals. We propose a pilot-assisted opportunistic user scheduling (PAOUS) scheme, consisting of low-complexity channel training, and one-bit feedback transmission. We com- 
pute the achievable rate region for the proposed scheme, and characterize the achievable degrees of freedom (DoF) region. Our results reveal that in a multi-cell network with $K$ base stations and a coherence time $T$, the achievable sum-rate increases as the number of mobile terminals scales. In addition, the sum DoF $K_{\text {opt }}\left(1-\frac{K_{\mathrm{opt}}}{T}\right)$ is achievable given that the number of mobile terminals in each cell scales proportional to signal-to-noise ratio (SNR). This result indicates that to maximize the achievable sum DoF only a subset of base stations should be activated, where the optimum number of active base stations is $K_{\text {opt }}=\min \left\{K, \frac{T}{2}\right\}$. The radio resources can be shared between channel training an data transmission phases. Using the computed achievable rate results, we numerically investigate the impact of power allocation between channel training and data transmission phases. Our study reveal that in large networks, which have many base stations, more power should be allocated to channel training while in dense networks, that have many mobile terminals in each cell, more power should be allocated for data transmission.

\section{Multi-Cell Multi-User Interference Network}

Consider downlink transmission in a network consisting $K$ neighbouring cells. In each cell, there is one single-antenna base station serving $N$ single-antenna mobile terminals. The base station in the $k$ th cell $(k \in\{1, \ldots, K\})$ is denoted as $\mathrm{BS}^{k}$, and the mobile terminals are shown as $\operatorname{MS}_{j}^{k}(j \in$ $\{1, \ldots, N\})$. Each base station intends to transmit independent messages to mobile terminals in the corresponding cell.

The channel gain between $\mathrm{BS}^{k}$ and $\mathrm{MS}_{j}^{p}(p \in\{1, \ldots, K\})$ at time $t^{\prime}$ is denoted as $h_{j, k}^{p}\left(t^{\prime}\right)$. We consider blockfading channel model with coherence time $T$, where channel gains are constant within one fading block, i.e. $h_{j, k}^{p}(n T+t)=h_{j, k}^{p}(n T) \quad(t \in\{1, \ldots, T-1\})$, and change to independent values across subsequent blocks. Channel gains have zero mean complex Gaussian distribution, i.e. $h_{j, k}^{p} \sim \mathcal{C N}(0,1)$, and are mutually independent across different users and cells. We assume that no a priori CSI is available at mobile terminals and base stations.

\section{PAOUS SCHEME}

The proposed PAOUS scheme at each fading block is conducted in three subsequent phases: channel training, feedback transmission, user scheduling, and data transmission phases as shown in Fig. 1. Forward transmission (pilot and data transmission) and reverse transmission (feedback transmission) are conducted in frequency division duplex (FDD) systems. In the forward transmission, within each fading block, $\alpha T$ time slots are allocated to channel training phase and $(1-\alpha) T$ time slots are left for data transmission phase where $\alpha(0<\alpha<1)$ is the channel sharing factor. In the following, we explain each phase in more details.

\section{A. Channel Training Phase}

We consider a pilot-assisted channel training scheme to acquire an estimation of local CSI (i.e. channel gains between base stations and the corresponding mobile terminal) at each mobile terminal. Channel training is performed in an orthogonal fashion in which the training period is divided into $K$ equal training slots each of which has the duration of $T_{\tau}$ $\left(T_{\tau}=\alpha T / K\right)$. Each base station transmits $T_{\tau}$ pilot symbols during one training slot and remains silent during other time slots as shown in Fig. 2. Then, each mobile terminal in the network estimates the gain of the corresponding link between the active base station and itself. Consider transmission at the $n$th fading block. The base station $\mathrm{BS}^{k}(k \in\{1, \ldots, K\})$ sends $T_{\tau}$ known pilot symbols as follows

$$
X_{\tau}^{k}(t)=\sqrt{P_{\tau}}, t \in \mathcal{T}_{n}^{k}
$$

where $\mathcal{T}_{n}^{k}=\left\{n T+(k-1) T_{\tau}+1, \ldots, n T+k T_{\tau}\right\}$. Consequently, the received signals at mobile terminal $\mathrm{MS}_{j}^{p}$ $(p \in\{1, \ldots, K\}, j \in\{1, \ldots, N\})$ are

$$
Y_{\tau, j}^{p}(t)=\sqrt{P_{\tau}} h_{j, k}^{p}(n T)+Z_{j}^{p}(t), t \in \mathcal{T}_{n}^{k},
$$

where $Z_{j}^{p}(t)$ is the receiver noise which has Gaussian distribution with power $N_{0}$, i.e. $Z_{j}^{p}(t) \sim \mathcal{C N}\left(0, N_{0}\right)$. The mobile terminal performs a minimum mean square error (MMSE) estimation of the channel gain $h_{j, k}^{p}(n T)$ as follows

$$
\tilde{h}_{j, k}^{p}(n T)=\frac{P_{\tau}}{N_{0}+T_{\tau} P_{\tau}} \sum_{t=n T+(k-1) T_{\tau}+1}^{n T+k T_{\tau}} Y_{\tau, j}^{p}(t) .
$$

The following equation holds

$$
h_{j, k}^{p}(n T)=\tilde{h}_{j, k}^{p}(n T)+\varepsilon_{j, k}^{p}(n T),
$$

where $\varepsilon_{j, k}^{p}(n T)$ denotes corresponding channel estimation error. The random variables $\varepsilon_{j, k}^{p}(n T)$ and $\tilde{h}_{j, k}^{p}(n T)$ are independent zero mean Gaussian distributed with variances $\sigma_{\varepsilon}^{2}$ and $1-\sigma_{\varepsilon}^{2}$, respectively, where

$$
\sigma_{\varepsilon}^{2}=\frac{1}{1+T_{\tau} P_{\tau} / N_{0}}
$$

At the end of the training phase, mobile terminal $\mathrm{MS}_{j}^{p}$ obtains the estimation of local CSI, i.e. $\tilde{h}_{j, k}^{p}(n T)(k \in$ $\{1, \ldots, K\})$. This noisy estimation of CSI can be used to compute the feedback signal as described in the next part.

\section{B. Feedback Transmission and User Selection Phase}

Each mobile terminal measures the strength of interference links and locally make a decision whether the strength of interference links are below a certain threshold. Then, it sends a one-bit feedback signal to the corresponding base station. This measure is computed based on estimated local CSI. Specifically, in the $n$th fading block, $\mathrm{MS}_{j}^{p}$ computes $\delta_{j}^{p}(n T)$ that is defined as follows

$$
\delta_{j}^{p}(n T) \triangleq \sum_{i=1, i \neq j}^{K}\left|\tilde{h}_{j, i}^{p}(n T)\right|^{2}
$$

Next, it sends a one-bit feedback signal defined as follows

$$
f_{j}^{p}(n T) \triangleq\left\{\begin{array}{ll}
1 & \delta_{j}^{p}(n T) \leq \epsilon_{\mathrm{th}} \\
0 & \delta_{j}^{p}(n T)>\epsilon_{\mathrm{th}}
\end{array},\right.
$$




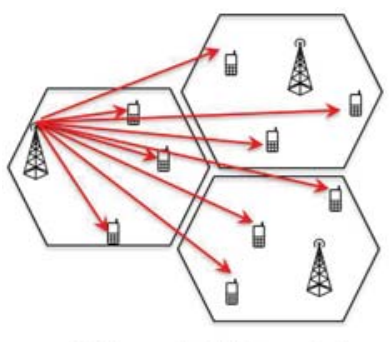

a. Orthogonal pilot transmission

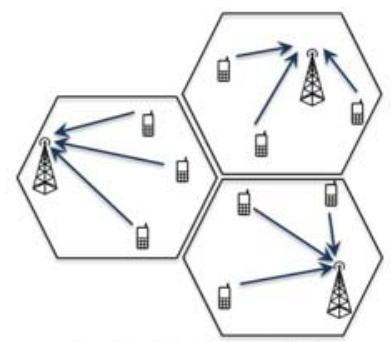

b. Feedback transmission

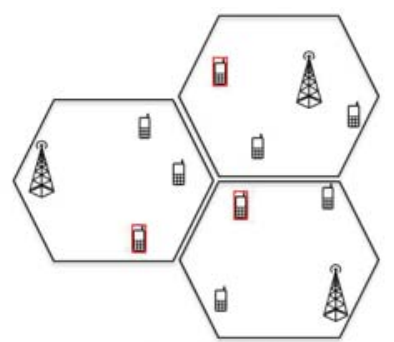

c. User scheduling

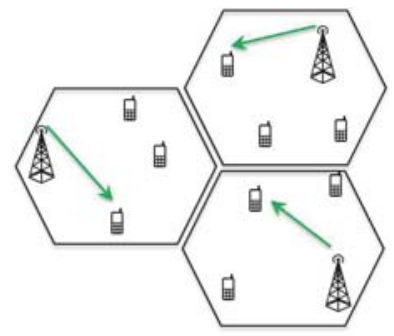

d. Data transmission

Fig. 1: Schematic representation of different phases of the proposed PAOUS scheme.

where $\epsilon_{\text {th }}$ is a positive constant. A smaller $\epsilon_{\text {th }}$ implies that lower interference is acceptable at mobile terminals with the cost of a lower probability to find such a mobile terminal. This is a design parameter that need to be optimized in order to maximize the achievable sum-rate. The feedback channels are orthogonal to each other. In practice, the feedback signals are also prone to errors. Since we intend to investigate the impact of channel estimation error on the performance of the transmission scheme we assume that feedback channels are error-free.

The base station $\mathrm{BS}^{k}$ collects feedback signals from all mobile terminals within the corresponding cell, i.e. $f_{j}^{k}(n T)$ $(j \in\{1, \ldots, N\})$. A mobile terminal is called a candidate mobile terminal to be scheduled if the corresponding feedback signal is one. We define the set of candidate mobile terminals in the $k$ th cell as follows

$$
\mathcal{A}^{k} \triangleq\left\{i \mid f_{i}^{k}(n T)=1, i \in\{1, \ldots, N\}\right\} .
$$

The number of candidate mobile terminals in the $k$ th cell is $\rho^{k}=\left|\mathcal{A}^{k}\right|$, where $|\mathcal{A}|$ is the cardinality of the set $\mathcal{A}$.

If $\rho^{k} \neq 0$, then $\mathrm{BS}^{k}$ schedules a randomly selected mobile terminal from the set of the candidate mobile terminals $\mathcal{A}^{k}$. The selected mobile terminal is denoted as $\mathrm{MS}_{\alpha_{k}}^{k}$. Otherwise, no mobile terminal will be scheduled. Since the network is symmetric, a random scheduling ensures that all mobile terminals will be scheduled with the same probability. This implies that the proposed scheme indeed offers fairness.

\section{Data Transmission Phase}

There are $N$ buffers at each base station, and each of them stores messages that should be sent to a specific mobile terminal. In the data transmission phase, each base station communicates to the scheduled mobile terminal in the associated cell. Message are encoded according to the multiplexed coding scheme similar to the one proposed in [12]. Corresponding to each mobile terminal, there are multiple codebooks each associated with a specific channel state. For a given channel state, $\mathrm{BS}^{k}(k \in\{1,2, \ldots, K\})$ selects message $m_{\alpha_{k}}^{k}$ independently with a uniform distribution from the set $\mathcal{M}=\left\{1,2, \ldots, 2^{N^{\prime} T_{d} \tilde{R}_{k}}\right\}$, where $\tilde{R}_{k}>0$ is the code rate, and $N^{\prime}$ is the number of fading blocks that span one codeword. Then, it encodes the message $m_{\alpha_{k}}^{k}$ to a length

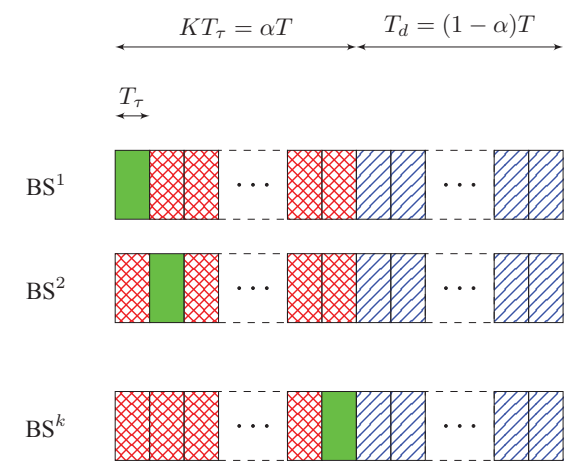

Fig. 2: Transmitted symbols by base stations $\mathrm{BS}^{k}$ $(k \in\{1, \ldots, K\})$ within one fading block. The crosshatched red slot, the plain green slot, and the blue angle lined slots denote no transmission, pilot symbols, and data symbols, respectively.

$N^{\prime} T_{d}$ codeword $\left\{X_{d, \alpha_{k}}^{k}(i)\right\}_{i=1}^{N^{\prime} T_{d}}$. Moreover, the codewords must satisfy a power constraint

$$
\mathbb{E}\left[\left|X_{d, \alpha_{k}}^{k}\right|^{2}\right]<P_{d} .
$$

In fading block $n, \mathrm{BS}^{k}$ sends $\left\{X_{d, \alpha_{k}}^{k}(i)\right\}_{i=n T+K T_{\tau}+1}^{(n+1) T}$ during $T_{d}$ data transmission time slots. All base stations transmit at the same time and the same frequency band. Consequently, the channel output at $\mathrm{MS}_{\alpha_{k}}^{k}$ is

$$
\begin{aligned}
Y_{d, \alpha_{k}}^{k}(i)= & h_{\alpha_{k}, k}^{k}(n T) X_{d, \alpha_{k}}^{k}(i) \\
& +\sum_{\substack{l=1, l \neq k \\
i}}^{K} h_{\alpha_{k}, l}^{k}(n T) X_{d, \alpha_{k}}^{l}(i)+Z^{k}(i), \\
& n T+K T_{\tau}+1, \ldots,(n+1) T
\end{aligned}
$$

where $Z^{k}(i) \sim \mathcal{C N}\left(0, N_{0}\right)$. The mobile terminal collects all $N^{\prime}$ received signals, decode the received codeword and estimate the transmitted message.

\section{Achievable Rate Region}

In this section, we study the achievable rate region of the proposed transmission scheme. 
Theorem 1. The base station-mobile terminal pair $\mathrm{BS}^{k}-\mathrm{MS}_{i}^{k}$ can achieve the following rate

$R_{i}^{k}=\left(1-K \frac{T_{\tau}}{T}\right) \gamma_{i}^{k} \mathbb{E}_{\tilde{h}}\left[\log \left(1+\frac{\beta|\tilde{h}|^{2} P}{N_{0}+\beta\left(K \sigma_{\varepsilon}^{2}+\epsilon_{\mathrm{th}}\right) P}\right)\right]$,

where

$$
\begin{aligned}
\sigma_{\varepsilon}^{2} & =\frac{1}{1+K T_{\tau}((1-(1-\alpha) \beta) / \alpha) P / N_{0}} \\
\gamma_{i}^{k} & =\frac{1}{N}\left(1-\left(1-F\left(\frac{\epsilon_{\mathrm{th}}}{\sigma_{\varepsilon}^{2}}\right)\right)^{N}\right) \\
F(x) & \triangleq \frac{\gamma\left(K-1, \frac{x}{2}\right)}{\Gamma(K-1)}
\end{aligned}
$$

and $\tilde{h} \sim \mathcal{C N}\left(0,1-\sigma_{\varepsilon}^{2}\right)$. The function $\Gamma(z) \triangleq \int_{0}^{\infty} t^{z-1} e^{-t} d t$ is the Gamma function, and $\gamma(z, x) \triangleq \int_{0}^{x} t^{z-1} e^{-t} d t$ is the lower incomplete Gamma function.

Proof. Assuming that $\mathrm{MS}_{\alpha_{k}}^{k}$ is scheduled, the mutual information between $\mathrm{BS}^{k}$ and the selected mobile terminal $\mathrm{MS}_{\alpha_{k}}^{k}$ can be lower bounded as follows

$$
\begin{aligned}
& I\left(X_{d, \alpha_{k}}^{k} ; Y_{d, \alpha_{k}}^{k} \mid \tilde{h}_{\alpha_{k}, 1}^{k}, \ldots, \tilde{h}_{\alpha_{k}, K}^{k}\right) \\
= & h\left(X_{d, \alpha_{k}}^{k} \mid \tilde{h}_{\alpha_{k}, 1}^{k}, \ldots, \tilde{h}_{\alpha_{k}, K}^{k}\right) \\
& -h\left(X_{d, \alpha_{k}}^{k} \mid \tilde{h}_{\alpha_{k}, 1}^{k}, \ldots, \tilde{h}_{\alpha_{k}, K}^{k}, Y_{d, \alpha_{k}}^{k}\right) \\
= & h\left(X_{d, \alpha_{k}}^{k}\right) \\
& -h\left(X_{d, \alpha_{k}}^{k}-\hat{X}_{d, \alpha_{k}}^{k} \mid \tilde{h}_{\alpha_{k}, 1}^{k}, \ldots, \tilde{h}_{\alpha_{k}, K}^{k}, Y_{d, \alpha_{k}}^{k}\right) \\
= & \log \left(2 \pi e P_{d}\right) \\
& -h\left(X_{d, \alpha_{k}}^{k}-\hat{X}_{d, \alpha_{k}}^{k} \mid \tilde{h}_{\alpha_{k}, 1}^{k}, \ldots, \tilde{h}_{\alpha_{k}, K}^{k}, Y_{d, \alpha_{k}}^{k}\right) \\
= & \log \left(2 \pi e P_{d}\right) \\
& -h\left(X_{d, \alpha_{k}}^{k}-\hat{X}_{d, \alpha_{k}}^{k} \mid \tilde{h}_{\alpha_{k}, 1}^{k}, \ldots, \tilde{h}_{\alpha_{k}, K}^{k}\right) \\
(a) & \log \left(2 \pi e P_{d}\right)-\log \left(2 \pi e \sigma^{2}\right), \\
\geq &
\end{aligned}
$$

where $\hat{X}_{d, \alpha_{k}}^{k} \triangleq f\left(\tilde{h}_{\alpha_{k}, 1}^{k}, \ldots, \tilde{h}_{\alpha_{k}, K}^{k}, Y_{d, \alpha_{k}}^{k}\right)$ is a function of the received signal and the estimated local CSI, and $\sigma^{2}$ is the variance of $\left(X_{d, \alpha_{k}}^{k}-\hat{X}_{d, \alpha_{k}}^{k}\right)$ given the estimated channel gains. In this equation $(a)$ follows the fact that the entropy of a random variable with a given variance is upper bounded with the entropy of a Gaussian distributed random variable. We select $\hat{X}_{d, \alpha_{k}}^{k}$ to be the MMSE estimate of $X_{d, \alpha_{k}}^{k}$ as follows

$$
\begin{aligned}
\hat{X}_{d}^{k} & =\frac{\mathbb{E}\left[\hat{X}_{d}^{k}\left(Y_{d, \alpha_{k}}^{k}\right)^{*} \mid \tilde{h}_{\alpha_{k}, 1}^{k}, \ldots, \tilde{h}_{\alpha_{k}, K}^{k}\right]}{\mathbb{E}\left[Y_{d, \alpha_{k}}^{k}\left(Y_{d, \alpha_{k}}^{k}\right)^{*} \mid \tilde{h}_{\alpha_{k}, 1}^{k}, \ldots, \tilde{h}_{\alpha_{k}, K}^{k}\right]} Y_{d, \alpha_{k}}^{k} \\
& =\frac{\left(\tilde{h}_{\alpha_{k}, k}^{k}\right)^{*} Y_{d, \alpha_{k}}^{k} P_{d}}{N_{0}+\left(K \sigma_{\varepsilon}^{2}+\epsilon_{\mathrm{th}}\right) P_{d}} .
\end{aligned}
$$

Therefore, the variance $\sigma^{2}$ in (12) is

$$
\begin{aligned}
\sigma^{2} & =\mathbb{E}\left[\left(X_{d}^{k}-\hat{X}_{d}^{k}\right)\left(X_{d}^{k}-\hat{X}_{d}^{k}\right)^{*} \mid \tilde{h}_{\alpha_{k}, 1}^{k}, \ldots, \tilde{h}_{\alpha_{k}, K}^{k}\right] \\
\stackrel{(a)}{=} & \mathbb{E}\left[X_{d}^{k}\left(X_{d}^{k}-\hat{X}_{d}^{k}\right)^{*} \mid \tilde{h}_{\alpha_{k}, 1}^{k}, \ldots, \tilde{h}_{\alpha_{k}, K}^{k}\right] \\
= & \frac{P_{d}}{N_{0}+\frac{\left|\tilde{h}_{\alpha_{k}, k}^{k}\right|^{2} P_{d}}{N_{0}+\left(K \sigma_{\varepsilon}^{2}+\epsilon_{\mathrm{th}}\right) P_{d}}}
\end{aligned}
$$

where $(a)$ follows the orthogonality principle of MMSE estimator. Substituting $\sigma^{2}$ in (12), the lower bound on mutual information can be computed.

In addition, the probability that the mobile terminal $\mathrm{MS}_{i}^{k}$ be scheduled is $\gamma_{i}^{k}=\frac{1}{N} \gamma^{k}$, where $\gamma^{k}$ is the probability that one mobile terminal be scheduled in the $k$ th cell. The probability $\gamma^{k}$ can be computed as follows

$$
\begin{aligned}
\gamma^{k} & =\operatorname{Pr}\left\{\bigcup_{i=1}^{N}\left\{\delta_{i}^{k}<\epsilon_{\mathrm{th}}\right\}\right\}=1-\operatorname{Pr}\left\{\bigcap_{i=1}^{N}\left\{\delta_{i}^{k}>\epsilon_{\mathrm{th}}\right\}\right\} \\
& =1-\prod_{i=1}^{N} \operatorname{Pr}\left\{\delta_{i}^{k}>\epsilon_{\mathrm{th}}\right\} \stackrel{(a)}{=} 1-\left(1-F\left(\frac{\epsilon_{\mathrm{th}}}{\sigma_{\varepsilon}^{2}}\right)\right)^{N},
\end{aligned}
$$

where $(a)$ follows the fact that the random variable $\frac{\delta_{i}^{k}}{\sigma_{\varepsilon}^{2}}$ has chi-square distribution with degrees of freedom $2(K-1)^{\varepsilon}$. The corresponding cumulative density function (CDF) is

$$
F(x)=\frac{\gamma\left(K-1, \frac{x}{2}\right)}{\Gamma(K-1)},
$$

where $\Gamma(z)=\int_{0}^{\infty} t^{z-1} e^{-t} d t$ is the Gamma function, and $\gamma(z, x)=\int_{0}^{x} t^{z-1} e^{-t} d t$ is the lower incomplete Gamma function.

Because of the energy conservation law we have

$$
P_{\tau} \alpha T / K+P_{d}(1-\alpha) T=P T,
$$

where

$$
\begin{aligned}
& P_{d}=\beta P \\
& P_{\tau}=K((1-(1-\alpha) \beta) / \alpha) P .
\end{aligned}
$$

The parameter $\beta(0 \leq \beta \leq 1 /(1-\alpha))$ is called power allocation factor. Substituting $P_{\tau}$ in (4), $\sigma_{\varepsilon}^{2}$ given in (10) can be computed.

Corollary 1. The achievable sum-rate of the network $\left(R_{\sum} \triangleq \sum_{k=1}^{K} \sum_{i=1}^{N} R_{i}^{k}\right)$ is

$R_{\sum}=K\left(1-K \frac{T_{\tau}}{T}\right) \gamma \mathbb{E}_{\tilde{h}}\left[\log \left(N_{0}+\frac{\beta|\tilde{h}|^{2} P}{1+\beta\left(K \sigma_{\varepsilon}^{2}+\epsilon_{\mathrm{th}}\right) P}\right)\right]$,

where

$$
\gamma=1-\left(1-F\left(\frac{\epsilon_{\mathrm{th}}}{\sigma_{\varepsilon}^{2}}\right)\right)^{N}
$$




\section{A. Achievable Sum Degrees of Freedom}

The achievable sum degrees of freedom, defined as the pre$\log$ factor of the achievable sum-rate in the asymptotically high-SNR regime, is characterized in the following theorem.

Theorem 2. The achievable sum degrees of freedom is

$$
d_{\sum}=K_{\mathrm{opt}}\left(1-\frac{K_{\mathrm{opt}}}{T}\right)
$$

where

$$
K_{\mathrm{opt}}=\min \left\{\frac{T}{2}, K\right\},
$$

if the number of mobile terminals in each cell $(N)$ scales proportional to SNR.

Proof. We set $\epsilon_{\text {th }}=1 / P$, and $N \propto P$. Then, the achievable sum degrees of freedom can be computed as $d_{\sum}=\lim _{P \rightarrow \infty} R_{\sum} / \log P$, where $R_{\sum}$ is given in Corollary $\mathbf{1}$. Using the dominated convergence theorem [13] it can be shown that this limit is equal to $K\left(1-\frac{K T_{\tau}}{T}\right)$. We select $T_{\tau}=1$ to maximize the achievable sum degrees of freedom. It can be shown that when $K>\frac{T}{2}$, the number of active base stations that maximizes the sum degrees of freedom is $K^{\prime}=\frac{T}{2}$.

To maximize the sum degrees of freedom, or equivalently the network throughput at high SNR regime, in large networks $(K>T / 2)$, Theorem 2 recommends to turn on only a subset of base stations, and perform the proposed PAOUS scheme within the cells with active base station. The intuition behind this result is that only limited radio resources are available for channel training and data transmission, and if in large networks we allocate orthogonal training slots to all base station then there may not be resources left for data transmission. Therefore, only a subset of base stations should be activated. In addition, this theorem crystalizes the dependency of the optimum number of active base stations on the channel coherence time. It also worth mentioning that since the network is symmetric a random base station selection will ensure fairness among users in different cells.

\section{B. Numerical Evaluation}

In this section, we numerically evaluate the performance of the proposed PAOUS scheme in sample communication networks. We consider three-cell network $(K=3)$ with $N$ mobile terminals in each cell, where $N$ can be possibly large in dense communication networks. Fig. 3 shows achievable sumrate versus SNR for different number of mobile terminals in each cell $(N)$. It can be seen that the sum-rate increases as $N$ increases. The reason is that as the number of mobile terminals increases, it is more likely that the set of candidate mobile terminals in (7) be nonempty and consequently a mobile terminal be scheduled in each cell. This in fact indicates that the proposed scheme in dense communication scenarios (many users in each cell) can effectively mitigate the inter-cell interference by properly selecting those mobile terminals that experience less interference.

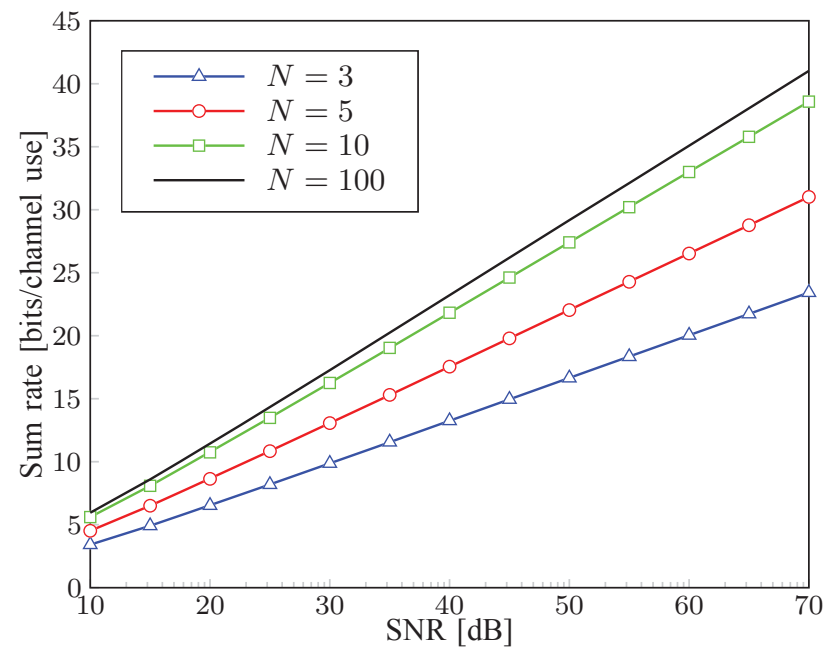

Fig. 3: Achievable sum-rate versus power for different number of mobile terminals in each cell $(N)$.

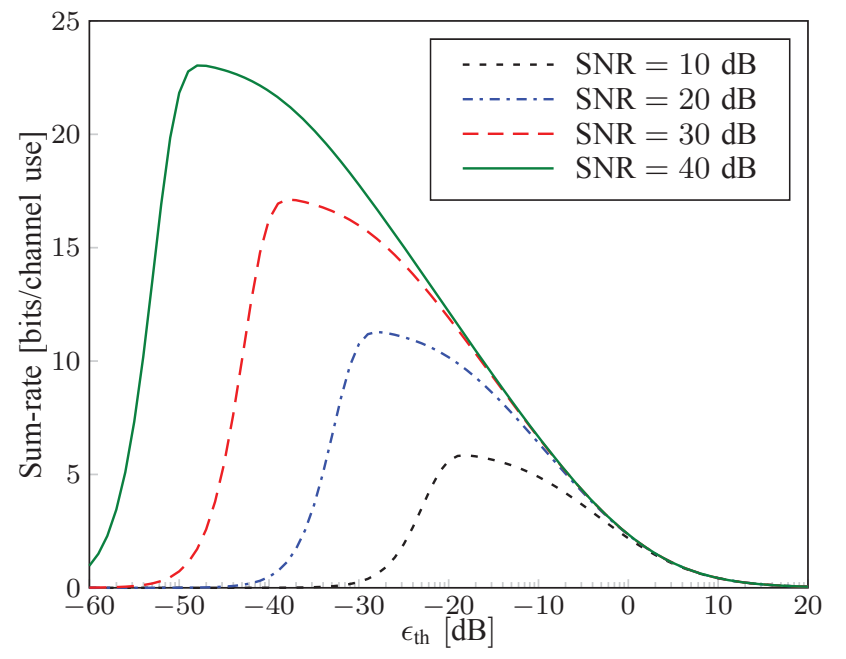

Fig. 4: Achievable sum-rate versus $\epsilon_{\mathrm{th}}$ for different SNR values.

Fig. 4 illustrates the achievable sum-rate as a function of threshold $\epsilon_{\text {th }}$ defined in (6) for different SNR values. It can be observed that, for a given SNR, a specific $\epsilon_{\text {th }}$ maximizes sumrate. The optimum $\epsilon_{\text {th }}$ decays as SNR increases. Increasing $\epsilon_{\text {th }}$ in one hand increases the probability that a mobile terminal be scheduled in each cell, on the other hand the corresponding mutual information decays as a consequence of a larger interference. In high-SNR regime where interference is dominant a smaller $\epsilon_{\text {th }}$ should be selected in order to limit the level of interference and increase the achievable sum-rate.

Fig. 5 shows the achievable sum-rate versus $\beta$ for different values of $N$. It can be observed that for each value of $N$ a specific $\beta\left(\beta_{\mathrm{opt}}\right)$ maximizes the achievable sum-rate. The optimum value of $\beta$ increases as $N$ increases. This implies that when there is a large number of mobile terminals in the network more power should be allocated to data transmission instead of channel training. 


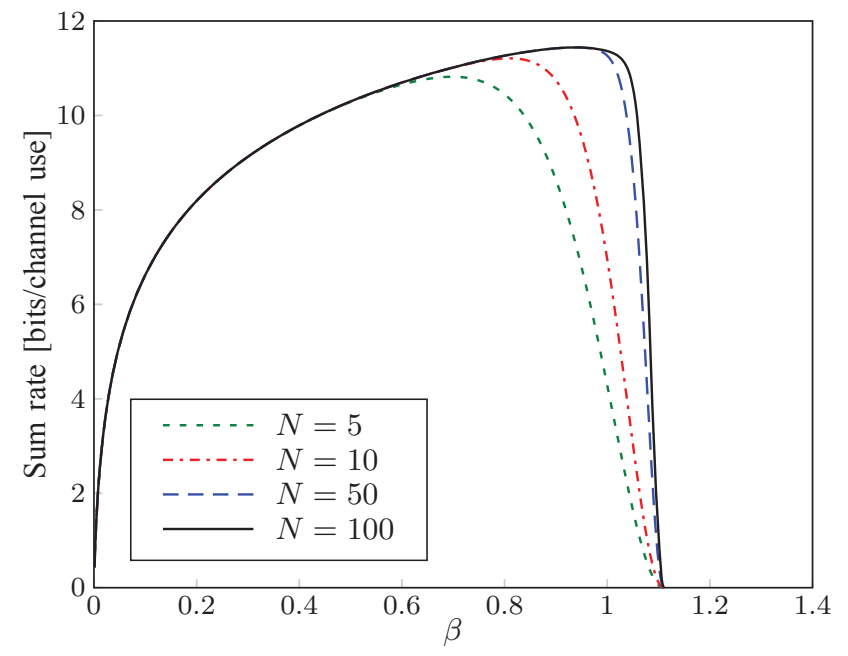

Fig. 5: Achievable sum-rate versus $\beta$ for different number of users in each cell $(N)$.

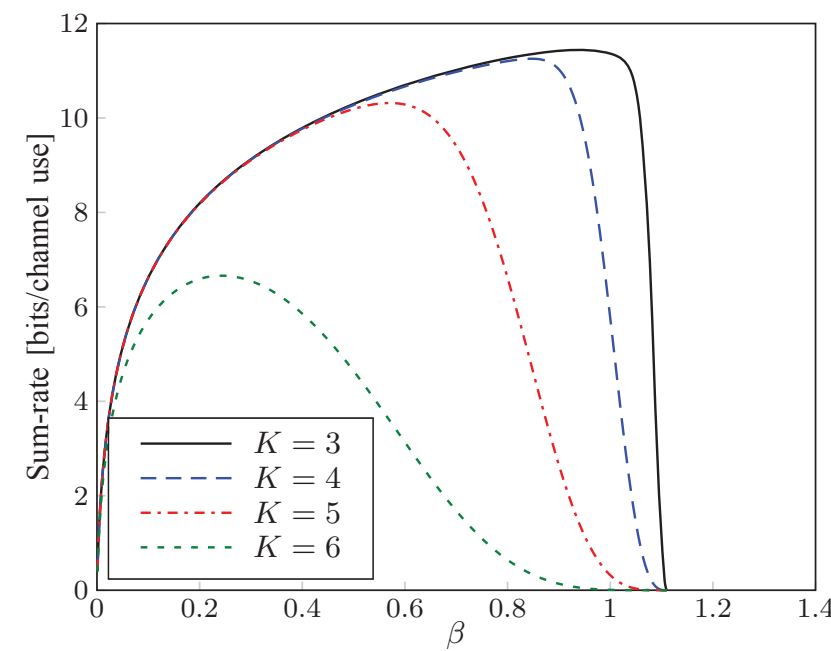

Fig. 6: Achievable sum-rate versus $\beta$ for different number of base stations $(K)$.

Fig. 6 shows the achievable sum-rate versus $\beta$ for different values of $K$. In this case also it can be seen that for each value of $K$ a specific $\beta$ maximizes the achievable sum-rate. The optimum value of $\beta$ decreases as $K$ increases. This implies that when there are many base stations in the network more power should be allocated to channel training instead of data transmission. The reason is that when the number of base stations increases, inter-cell interference become more severe and more accurate channel estimation is required for an effective user scheduling.

\section{CONCLUSION}

In this paper, we have investigated a typical scenario in $5 \mathrm{G}$ communication systems, where a large number of users in a multi-cell multi-user network have to be served efficiently (e.g. with low training and feedback overhead), when no $a$ priori CSI is available at terminals. We proposed a pilot- assisted opportunistic user scheduling (PAOUS) scheme, and showed that the proposed scheme is well-suited for such scenarios: it offers low-computational complexity, and requires only a one-bit feedback signal from mobile terminals to their respective base stations to perform scheduling. Furthermore, we computed the achievable rate region for the proposed scheme. We have shown that the achievable sum-rate scales as the number of mobile terminals in each cell increases. Our results reveal that in a multi-cell network with $K$ base stations, given that the number of mobile terminals in each cell properly scales with SNR, the sum DoF $K_{\text {opt }}\left(1-\frac{K_{\text {opt }}}{T}\right)$ is achievable, where $K_{\text {opt }}=\min \left\{K, \frac{T}{2}\right\}$ is the optimum number of the base stations that need to be activated in the network. We have also investigated the problem of power allocation between channel training and data transmission phases. Our numerical evaluations reveal that in large networks (many base stations) more power should be allocated to channel training, while in dense networks (many mobile terminals in each cell) more power should be allocated to data transmission instead.

\section{REFERENCES}

[1] A. Osseiran, F. Boccardi, V. Braun, K. Kusume, P. Marsch, M. Maternia, O. Queseth, M. Schellmann, H. Schotten, H. Taoka, H. Tullberg, M. A. Uusitalo, B. Timus, and M. Fallgren, "Scenarios for $5 \mathrm{G}$ mobile and wireless communications: the vision of the METIS project," IEEE Commun. Mag., vol. 52, no. 5, pp. 26-35, May 2014.

[2] L. Xin, E. K. P. Chong, and N. B. Shroff, "Opportunistic transmission scheduling with resource-sharing constraints in wireless networks," IEEE Journal on Selected Areas in Communications, vol. 19, no. 10, pp. 2053-2064, Oct. 2001.

[3] D. Liang, L. Teng, and H. Yih-Fang, "Opportunistic transmission scheduling for multiuser MIMO systems," in IEEE Int. Conf. Acoustics, Speech, and Signal Processing (ICASSP'03), Hong Kong, Hong Kong, Apr. 2003.

[4] A. Tajer and X. Wang, "Opportunistic multi-antenna downlink transmission with finite-rate feedback," in Annual Allerton Conference on Communication, Control, and Computing, 2008.

[5] M. A. Sadrabadi, A. Bayesteh, and E. Modiano, "Opportunistic scheduling in large-scale wireless networks," in IEEE Int. Symp. Information Theory (ISIT'09), Seoul, Korea, 2009.

[6] P. Viswanath, D. N. C. Tse, and R. Laroia, "Opportunistic beamforming using dumb antennas," IEEE Trans. Inf. Theory, vol. 48, no. 6, pp. 12771294, Aug. 2002.

[7] M. Sharif and B. Hassibi, "On the capacity of MIMO broadcast channels with partial side information," IEEE Trans. Inf. Theory, vol. 51, no. 2, pp. 506-522, Feb. 2005.

[8] S. M. Perlaza, M. Debbah, S. Lasaulce, and J. M. Chaufray, "Opportunistic interference alignment in MIMO interference channels," in IEEE Int. Symp. on Personal, Indoor and Mobile Radio Communications (PIMRC'08), Cannes, France, Sep. 2008.

[9] B. C. Jung, G. Nat, D. Park, and W. Y. Shin, “Opportunistic interference mitigation achieves optimal degrees-of-freedom in wireless multi-cell uplink networks," IEEE Trans. Commun., vol. 60, no. 7, pp. 1935-1944, Jul. 2012.

[10] H. J. Yang, W. Y. Shin, B. C. Jung, and C. Suh, "Opportunistic interference alignment for MIMO interfering broadcast channels," in IEEE Int. Conf. on Acoustics, Speech and Signal Processing (ICASSP'14), Florence, Italy, May 2014.

[11] H. Liu, H. Gao, W. Long, and T. Lv, "A novel scheme for downlink opportunistic interference alignment," arxiv.org, 2014.

[12] A. J. Goldsmith and P. P. Varaiya, "Capacity of fading channels with channel side information," IEEE Trans. Inf. Theory, vol. 43, no. 6, pp. 1986-1992, Nov. 1997.

[13] J. McDonald and N. A. Weiss, A course in real analysis, 2nd ed. Elsevier Inc., 2012. 